\title{
Evaluación de las pérdidas laborales provocadas enfermedades y lesiones en España en el año 2009
}

\section{Assessment of productivity losses due to diseases and injuries in Spain in 2009}

Núm. 6 (2016), pp. 66-85

Peña Longobardo, Luz María*1

Aranda Reneo, Isaac ${ }^{* 2}$

Oliva-Moreno, Juan*3

Vall Castello, Judit*4

Recibido: diciembre, 2014

Aceptado: enero, 2016

JEL Clasif: Jo1;J24;I18

DOI: $10.5944 /$ reppp.6.2016.13841

\footnotetext{
*1 Luz María Peña Longobardo. Investigadora FPU-Departamento de Análisis EconómicoUniversidad de Castilla la Mancha.E-mail: LuzMaria.Pena@uclm.es

*2 Isaac Aranda Reneo. Profesor Contratado Doctor Temporal- Departamento de Análisis EconómicoUniversidad de Castilla La Mancha. E-mail: Isaac.Aranda@uclm.es

*3 Juan Oliva Moreno. Universidad de Castilla La Mancha. Departamento de Análisis Económico y Finanzas. Editor Asociado. E-mail: juan.olivamoreno@uclm.es

*4 Judit Vall Castello. Investigadora en el Centro de Investigación en Economía y Salud (Universidad Pompeu Fabra). E-mail: judit.vall@upf.edu
} 


\title{
Resumen
}

El objetivo del trabajo es estimar las potenciales pérdidas laborales ocasionadas por enfermedades y lesiones durante el año 2009. Combinando varias fuentes de datos del Instituto Nacional de Estadística y el Instituto Nacional de la Seguridad Social, se desarrolla un modelo de simulación basado en el enfoque del capital humano para la estimación de pérdidas de producción asociadas a muertes prematuras y a situaciones de incapacidad laboral (permanente y temporal).

Las pérdidas potenciales estimadas ascienden a 42.430 millones de euros, 8.073 millones de euros corresponden a pérdidas derivadas de la mortalidad prematura (19,0\% sobre el total), 18.050 millones de euros corresponden a pérdidas asociadas a situaciones de Incapacidad Permanente (42,5\% sobre el total) y 16.306 millones de euros a pérdidas asociadas a situaciones de Incapacidad Temporal (38,4\% sobre el total). Ello supone una cifra equivalente al 3,93\% del Producto Interior Bruto de España o, alternativamente, al $60,3 \%$ del gasto sanitario público total del año 2009. Por tipo de enfermedad o lesión, las enfermedades del sistema osteomuscular y del tejido conjuntivo representaron el 22,9\% de las pérdidas, seguidos de las causas externas, con un 13,5\%, los trastornos mentales, $10,5 \%$, y los tumores, con un $10,4 \%$.

Palabras clave: pérdidas laborales; coste de la enfermedad; impacto laboral

\begin{abstract}
The main aim of this study is to estimate the productivity losses caused by diseases and injuries occurred in Spain in 2009. Using several data sources from the National Statistics Institute and The National Institute of Social Security, a simulation model based on the human-capital approach is performed to estimate the labour losses due to premature deaths and disability (permanent and temporary).

The total productivity losses are estimated to amount to 42,430 million of euros, of which 8,073 million of euros are due to the losses caused by premature deaths (19\% of the total loss), 18,050 million of euros to permanent disability ( $42 \%$ of the total loss) and 16,306 million of euros to temporary disability (38.4\% of the total loss). These figures represented 3.93\% of the 2009 Gross Domestic Product of Spain, in other words, 60.3\% of the total Public Health Expenditure in 2009. By type of disease or injury, diseases of musculoskeletal system and connective tissue accounted for $22.9 \%$ of the total loss, followed by external causes with $13.5 \%$, metal disorders with $10.5 \%$ and tumors with $10.4 \%$.
\end{abstract}

Key Words: productivity losses; cost of illness; labour impact 


\section{Introducción}

La salud es uno de los pilares donde se apoya el bienestar de los individuos y las sociedades. Desde la óptica económica, la salud es un bien de naturaleza dual. En primer lugar, es un bien valorado por la propia utilidad o bienestar que directamente aporta. Aunque disfrutar de una buena salud no es condición suficiente para alcanzar o mantener un alto grado de bienestar y felicidad, en términos generales sí es condición necesaria, como se ha demostrado en diversos trabajos sobre determinantes de la felicidad y satisfacción con la vida (Blanchflower et al., 2011; Ferrer-i-Carbonell, 2013; Graham, 2008). En segundo lugar, la salud es un componente fundamental del capital humano de las personas y las naciones. Así, inversiones en la mejora de la salud de las personas se puede traducir en mejoras en el rendimiento escolar y en la productividad laboral, lo cual redundará a su vez, en mejores posibilidades personales y de mayor desarrollo económico (Grossman, 1972b, 2000). Por tanto, en su faceta de bien de inversión, el que una sociedad disfrute de un buen estado de salud no garantiza su progreso económico futuro pero sí es un requisito esencial para ello (Sachs, 2001; Suhrcke et al., 2006).

La literatura científica sobre los estudios de coste de la enfermedad ha crecido de manera considerable en los últimos años (Akobundu et al., 2006; Beck et al., 2010; Clabaugh et al., 2008; Hu, 2006; Ng et al., 2014; Withrow et al., 2011). Estos estudios tratan de informar sobre el impacto económico ocasionado por una enfermedad o lesión en una población dada en un momento concreto del tiempo (enfoque de prevalencia) o trata de modelizar la evolución futura de un grupo de personas o pacientes afectados por un determinado problema de salud (enfoque de incidencia). Además de los recursos invertidos en la atención sanitaria para la prevención y el tratamiento de estos problemas, cada vez es más frecuente la inclusión de otros recursos cuyo uso habitual se ve modificado por el curso de las enfermedades y lesiones. La consideración explícita de estos recursos no sanitarios ayuda a una mayor comprensión del impacto económico que ocasionan determinados problemas de salud, complementando a su vez a otros indicadores que remiten a la pérdida directa de bienestar como son la mortalidad, morbilidad, discapacidad y pérdidas en calidad de vida ocasionadas tanto en pacientes como en familiares.

Sin embargo, aunque la presencia de los costes no sanitarios en la literatura está cada vez más presente (Hoefman et al., 2013; Krol et al., 2013), apenas existen trabajos que hayan tratado de estimar el impacto laboral sobre una determinada región o país de todas las enfermedades y lesiones en su conjunto. Los escasos trabajos realizados sobre el tema nos remiten al estudio publicado por el Minister of Public Works and Government Services Canada (2002), donde se estimó que las pérdidas laborales ocasionadas por enfermedades y lesiones podrían representar una cifra similar al 4,4\% del PIB canadiense (año de referencia 1998), así como los trabajos desarrollados por National Heart, Lung and Blood Institute (EE.UU), donde la última estimación disponible señala una pérdida laboral cercana al 4,3\% del PIB estadounidense (año de referencia 2009). En Europa no tenemos constancia de este tipo de estimaciones, con la excepción de España donde dos trabajos previos han estimado, primero para el año 2005, las pérdidas laborales ocasionadas por enfermedades y lesiones en cifras cercanas al 4,2\% del PIB (Oliva-Moreno, 2012) y, a continuación para el año 2007, en cifras cercanas al 4,0\% del PIB (Oliva et al., 2012). 
El objetivo primordial de este trabajo es continuar con la serie de estimaciones realizadas en años previos, mejorando la metodología y las fuentes de datos empleadas, para realizar una estimación de las pérdidas laborales ocasionadas por enfermedades y lesiones tomando como año de referencia el 2009.

Mientras el primer trabajo (estimación para el año 2005) (Oliva-Moreno, 2012) contaba con la ventaja de su originalidad, el segundo trabajo (Oliva et al., 2012) mejoraba los métodos y ampliaba las fuentes de datos al utilizar la Muestra Continua de Vidas Laborales para controlar el efecto de personas con Incapacidad Permanente (IP) que continúan trabajando y emplear una base de datos proporcionada por el Instituto Nacional de la Seguridad Social (INSS) para estimar la distribución por causa de enfermedad de bajas y pérdidas laborales asociadas a la Incapacidad Temporal (IT), beneficiándose adicionalmente de mejoras en la gestión de la información de las bajas por IT desarrolladas recientemente por el INSS. En este tercer trabajo nos apoyamos en la investigación desarrollada por colegas del campo de la epidemiología y la salud pública (Lopez et al., 2014) para añadir la distribución de enfermedades causantes de las bajas por IP. Así pues, se trata de un trabajo de actualización y continuación que amplía y mejora la información de trabajos previos en dos puntos clave: por un lado, incorpora información sobre la distribución de IP por causa de enfermedad o lesión procedente del trabajo de López et al.; por otro lado, tomando como base las anteriores estimaciones, permite tener un panorama mucho más completo de la distribución del impacto laboral teniendo en cuenta la enfermedad o lesión (tanto en la lista de grupos amplios como en la lista reducida de enfermedades con mayor impacto económico). Esta información añadida puede ser de gran utilidad para los decisores públicos en el diseño, planificación y evaluación de sus políticas de salud.

Tras este primer epígrafe introductorio, el segundo epígrafe refiere la metodología y las bases de datos empleadas en los modelos de simulación de pérdidas laborales. A continuación, en el tercer epígrafe, mostramos los principales resultados, en los tres componentes principales señalados y considerando su distribución regional y por tipo de enfermedad o lesión asociada a la pérdida. En el cuarto epígrafe discutimos los resultados encontrados, señalando las principales limitaciones del estudio pero también sus implicaciones para el diseño, implementación y evaluación de políticas y finalizamos con unas breves conclusiones.

\section{Material y métodos}

Las pérdidas de productividad ocasionadas por enfermedades y lesiones valoradas en términos monetarios se estimaron tanto para el caso de fallecimientos prematuros (antes de la edad de jubilación) como para bajas laborales temporales y permanentes. Dada la imposibilidad de medir directamente la productividad laboral perdida, nos basamos en las teorías económicas del capital humano (Becker, 1964; Grossman, 1972a, 2000; Hodgson et al., 1982; Max et al., 1990) para considerar que el salario bruto es la variable que permite medir y valorar de una forma razonable la productividad del trabajador. Para obtener el salario bruto de un trabajador medio se utilizó la Encuesta de Estructura Salarial (EES) elaborada por el Instituto Nacional de Estadística (INE) en 2009, la cual nos permitió ajustar el salario según la edad, el sexo y la Comunidad Autónoma de residencia (INE, 2009a). 
Además de la valoración monetaria, para la evaluación del impacto de las enfermedades y lesiones se utilizaron unidades físicas. En el caso de los fallecimientos prematuros se emplearon los Años Potenciales de Vida Laboral Perdidos (APVLP); en el caso de las bajas laborales temporales se identifican el número de casos y los días de baja y, para las situaciones de IP, el número de casos. Estos indicadores nos aportan información complementaria sobre la carga de las enfermedades y lesiones y son una primera aproximación sobre el impacto económico que éstas generan.

Para obtener el número de fallecimientos se utilizó el Registro de Defunciones según la causa de la muerte que elabora el INE. En este registro se encuentran los fallecimientos producidos en el territorio nacional y la causa que los originó (INE, 2009c). Se utilizaron los datos correspondientes a personas residentes en España que hubieron fallecido durante 2009. Se obtuvo la edad, el sexo, la Comunidad Autónoma de residencia y la causa que originó el fallecimiento, codificada mediante la Clasificación Internacional de Enfermedades de la Organización Mundial de la Salud (10 a revisión).

Una vez conocida la edad de fallecimiento de cada persona se estimó el flujo presente y futuro de la productividad laboral perdida por cada muerte prematura ocasionada por cualquiera de las causas consideradas. Con este fin, para cada muerte producida en un grupo de edad y sexo, se aplicaron las tasas de ocupación y los salarios esperados en cada periodo posterior al fallecimiento hasta un límite prefijado (en nuestro caso, los 65 años). Para ello se utilizó la tasa de ocupación para el 2009 que ofrece la Encuesta de Población Activa (EPA) elaborada por el INE, ajustando por edad, sexo y Comunidad Autónoma (INE, 2009b). La tasa de ocupación o tasa de empleo se define como el porcentaje de la población ocupada en relación con la población en edad de trabajar. A los valores futuros obtenidos, se les aplicó una tasa anual de descuento del tres por ciento y una tasa anual de crecimiento de la productividad laboral del uno por ciento. Este resultado se consideró como un «escenario base», al cual se aplicó un análisis de sensibilidad que incluyó dos tasas de descuento alternativas: cero por ciento y seis por ciento; y dos nuevas tasas de crecimiento de la productividad laboral: cero por ciento y dos por ciento. La tasas de descuento empleadas son habituales en ejercicios de evaluación económica en el campo de la salud (López-Bastida et al., 2010). En cuanto al crecimiento de la de productividad, se escogieron diferentes valores para representar diferentes escenarios económicos futuros: $1 \%$ representaría un crecimiento moderado de la productividad; $\mathrm{o} \%$ un crecimiento de productividad nulo y $2 \%$ un crecimiento de la productividad vigorosa.

Asimismo, se estimaron los APVLP a consecuencia de los fallecimientos prematuros. Para ello, se consideró como edad límite de permanencia en el mercado de trabajo, la edad legal de jubilación en España durante el año 2009, es decir, los 65 años. En el caso de los fallecimientos de personas que aún no habían alcanzado la edad mínima de participación laboral, los APVLP se estiman a partir de la edad legal de entrada al mercado laboral (es decir, se empieza a contabilizar desde los 16 años).

En el caso de las pérdidas de productividad ocasionadas por enfermedades y lesiones no mortales, se utilizaron datos sobre IT e IP recogidos en el Anuario de Estadísticas Sociales, publicado por el Ministerio de Trabajo y Asuntos Sociales. En el caso de las IP, los datos ofrecidos por el Ministerio de Trabajo incluyen el número de casos de IP, distinguiendo por el tipo de incapacidad (gran invalidez, incapacidad total e incapacidad absoluta) según la Comunidad Autónoma de residencia, el sexo y la edad. No obstante, 
en España las personas con la declaración de incapacidad absoluta y total pueden desempañar un trabajo remunerado. Para ajustar los datos brutos de IP y considerar que un porcentaje de esta población continúa en activo, utilizamos la información contenida en la Muestra Continua de Vida Laboral (MCVL) para obtener las tasas de empleo en personas con este tipo de IP (total o absoluta) y ajustar así nuestras estimaciones. Una vez se estima el número de casos de IP ajustado, la traducción de las unidades físicas (número de casos de IP) a cifras monetarias, se realizó aplicando los salarios brutos obtenidos de la EES.

Por otro lado, en las fuentes estadísticas disponibles no consta la enfermedad o lesión que origina o es causa principal de la baja permanente (IP). Para cubrir esta laguna, recurrimos al reciente trabajo realizado por López et al. (2014), quienes conectan información sobre el inicio de las IP (inicialmente sin el diagnóstico médico) con información de los registros de IT del año 2009 procedentes del Instituto Nacional de la Seguridad Social (INSS) que sí recoge el diagnóstico de cada episodio. Tras un proceso de depuración de datos, los autores alcanzan una aproximación a la distribución de IP por causa de enfermedad o lesión.

Finalmente, los datos empleados para la estimación de las pérdidas laborales asociadas a situaciones de IT, es decir, aquella situación del trabajador en la que se encuentra imposibilitado temporalmente para trabajar y precisa asistencia sanitaria, se obtuvieron de los registros del Instituto Nacional de la Seguridad Social. Dicha información, referida a la duración media de los procesos finalizados en el periodo y al número medio mensual de procesos iniciados en el periodo. Estos datos agregados se complementan con la información procedente de una muestra de 250.000 expedientes anonimizados de IT referidos al año 2009 tramitados por el INSS y facilitados para la realización de este trabajo. Esta muestra aporta información sobre la duración de las bajas, la estructura por edades y la causa médica de las IT para cada Comunidad Autónoma. La traducción de las unidades físicas (número de días de bajas por IT) a cifras monetarias, se realizó aplicando los salarios brutos obtenidos de la EES.

La perspectiva utilizada es la social, con un enfoque mixto: enfoque de incidencia para la mortalidad y enfoque de prevalencia para la IT y la IP. Este enfoque mixto es empleado con frecuencia en la literatura de estudios de coste de la enfermedad y facilita la comparabilidad entre estudios.

\section{Resultados}

El número de fallecimientos acaecidos en España durante el año 2009 ascendió a 383.209 , de los cuales un $52 \%$ correspondieron a varones y un $48 \%$ a mujeres. Esta proporción fue diferente en el caso de las muertes en edad laboral, 69\% varones y $31 \%$ mujeres de un total de 62.558 fallecimientos. Tres grupos de enfermedades (tumores, enfermedades del sistema circulatorio y enfermedades del sistema respiratorio) fueron responsables del 70\% de los fallecimientos. Por su parte, los tres grupos de enfermedades que mayor impacto produjeron en términos de APVLP fueron tumores (33\%), causas externas de mortalidad (19\%) y las enfermedades del sistema circulatorio (13\%). En su conjunto, estas tres causas de enfermedad fueron responsables de un $62 \%$ del total de fallecimientos acaecidos y un 66\% de los APVLP. 
Cuadro 1: Principales indicadores derivados de los fallecimientos, valores absolutos y distribución según causa del fallecimiento, 2009.

\begin{tabular}{|c|c|c|c|c|c|c|}
\hline & \multicolumn{2}{|c|}{ Fallecimientos } & \multicolumn{2}{|c|}{$\begin{array}{c}\text { Fallecimientos en } \\
\text { menores de } 65 \text { años }\end{array}$} & \multicolumn{2}{|c|}{ APVLP } \\
\hline & Total & Porcentaje & Total & Porcentaje & Total & Porcentaje \\
\hline Enfermedades infecciosas y parasitarias ${ }^{(1)}$ & 6.813 & $1,78 \%$ & 1.984 & $3,17 \%$ & 36.685 & $4,04 \%$ \\
\hline Tumores & 104.918 & $27,38 \%$ & 27.241 & $43,55 \%$ & 298.753 & $32,87 \%$ \\
\hline Enf. de sangre y de órg. hematopoyéticos & 1.456 & $0,38 \%$ & 194 & $0,31 \%$ & 3.889 & $0,43 \%$ \\
\hline Enfermedades endocrinas, nutricionales y metabólicas & 12.417 & $3,24 \%$ & 1.151 & $1,84 \%$ & 15.919 & $1,75 \%$ \\
\hline Trastornos mentales y del comportamiento & 14.451 & $3,77 \%$ & 440 & $0,70 \%$ & 5.585 & $0,61 \%$ \\
\hline Enfermedades del sistema nervioso y de los órganos de los sentidos & 18.748 & $4,89 \%$ & 1.673 & $2,67 \%$ & 31.197 & $3,43 \%$ \\
\hline Enfermedades del sistema circulatorio & 119.366 & $31,15 \%$ & 10.775 & $17,22 \%$ & 123.150 & $13,55 \%$ \\
\hline Enfermedades del sistema respiratorio & 43.178 & $11,27 \%$ & 3.077 & $4,92 \%$ & 42.620 & $4,69 \%$ \\
\hline Enfermedades del sistema digestivo & 19.460 & $5,08 \%$ & 3.938 & $6,29 \%$ & 47.925 & $5,27 \%$ \\
\hline Enfermedades de la piel y del tejido subcutáneo & 1.194 & $0,31 \%$ & 56 & $0,09 \%$ & 694 & $0,08 \%$ \\
\hline Enfermedades del sistema osteomuscular y del tejido conjuntivo & 3.451 & $0,90 \%$ & 204 & $0,33 \%$ & 2.813 & $0,31 \%$ \\
\hline Enfermedades del sistema genitourinario & $10.83^{8}$ & $2,83 \%$ & 488 & $0,78 \%$ & $5 \cdot 547$ & $0,61 \%$ \\
\hline Embarazo, parto y puerperio & 17 & $0,00 \%$ & 17 & $0,03 \%$ & 556 & $0,06 \%$ \\
\hline Afecciones originadas en el periodo perinatal & 884 & $0,23 \%$ & 882 & $1,41 \%$ & 43.040 & $4,73 \%$ \\
\hline Malformaciones congénitas, deformidades y anomalías cromosómicas & 875 & $0,23 \%$ & 777 & $1,24 \%$ & 29.815 & $3,28 \%$ \\
\hline Síntomas, signos y hallazgos anormales clínicos y de laboratorio, $\mathrm{NCOP}^{(1)}$ & 10.966 & $2,86 \%$ & 2.352 & $3,76 \%$ & 44.826 & $4,93 \%$ \\
\hline Causas externas de mortalidad & 14.177 & $3,70 \%$ & 7.309 & $11,68 \%$ & 175.988 & $19,36 \%$ \\
\hline Todas las causas & 383.209 & $100,00 \%$ & 62.558 & $100,00 \%$ & 909.002 & $100,00 \%$ \\
\hline
\end{tabular}

(1) Por motivos de comparabilidad, el Registro de Defunciones incluye el VIH+ (R75) en el Capítulo I. Enfermedades infecciosas y parasitarias aunque la CIE-1o lo encuadra en el Capítulo XVIII (Síntomas, signos y hallazgos anormales clínicos y de laboratorio, NCOP). Fuente: elaboración propia a partir del Registro de Defunciones por Causa de Muerte (año 2009). 
Por su parte, las pérdidas laborales ocasionadas por muertes prematuras en España en el año 2009 (aplicando el escenario base) se estimaron, en 8.073 millones de euros Dos grupos de enfermedades destacan sobre el resto y conjuntamente suponen más del 50\% de las pérdidas laborales asociadas a fallecimientos prematuros: tumores, con 2.422 millones de euros (el 30\% del total) y causas externas de mortalidad con 1.782 millones de euros (el 22\% sobre el total). Las enfermedades del sistema circulatorio aparecen en tercer lugar, representando un coste de 1.131 millones de euros (el 14\% sobre el total). La edad media de fallecimiento en el caso de las enfermedades del sistema circulatorio fue mayor que en el caso de tumores y causas externas (81 años en enfermedades de sistema circulatorio frente a 72 años en tumores y 60 en causas externas). De ahí que su impacto, en términos de APVLP y pérdidas laborales sean notoriamente inferiores aun siendo la primera causa de muerte en 2009. Los tres siguientes grupos de mayor impacto sobre las pérdidas de producción estimadas fueron las enfermedades del sistema digestivo (451 millones de euros), junto con síntomas, signos y hallazgos anormales clínicos (429 millones de euros) y las enfermedades del sistema respiratorio (383 millones de euros).

$\mathrm{Si}$ acudimos a la lista reducida de enfermedades (Clasificación Internacional de Enfermedades- $10^{a}$ revisión), 9 tipos de enfermedades (SIDA; tumor colon; tumor tráquea, bronquios y pulmón; tumor de mama; infarto agudo de miocardio; enfermedad cerebrovascular; cirrosis y otras enfermedades del hígado; suicidios y lesiones autoinfligidas; y accidentes de tráfico) causaron en 2009 el 38,55\% del total de fallecimientos de menos de 65 años (24.119 muertes), el 35,63\% del total APVLP (323.847 APVLP) y el $36,59 \%$ del total de las pérdidas laborales (cerca de 3.000 millones de euros). 
Cuadro 2: Pérdidas laborales ocasionadas por muerte prematura, valores absolutos y distribución según causa del fallecimiento.

\begin{tabular}{|c|c|c|c|c|c|c|}
\hline & \multicolumn{2}{|c|}{ Escenario base } & \multicolumn{2}{|c|}{ Escenario 1 } & \multicolumn{2}{|c|}{ Escenario 2 } \\
\hline & Miles de $€$ & $\%$ & Miles de $€$ & $\%$ & Miles de $€$ & $\%$ \\
\hline Enfermedades infecciosas y parasitarias & 365.736 & $4,53 \%$ & 550.845 & $4,25 \%$ & 275.201 & $4,61 \%$ \\
\hline Tumores & 2.422 .526 & $30,01 \%$ & 3.353 .801 & $25,87 \%$ & 1.940 .893 & $32,49 \%$ \\
\hline Enfermedades endocrinas, nutricionales y metabólicas & 134.055 & $1,66 \%$ & $223 \cdot 316$ & $1,72 \%$ & 96.817 & $1,62 \%$ \\
\hline Trastornos mentales y del comportamiento & 52.392 & $0,65 \%$ & 75.949 & $0,59 \%$ & 40.861 & $0,68 \%$ \\
\hline Enf. del sistema nervioso y de los órganos de los sentidos & 261.598 & $3,24 \%$ & 484.143 & $3,73 \%$ & 175.647 & $2,94 \%$ \\
\hline Enfermedades del sistema respiratorio & 383.923 & $4,76 \%$ & 599.091 & $4,62 \%$ & 288.973 & $4,84 \%$ \\
\hline Enfermedades del sistema digestivo & 451.799 & $5,60 \%$ & 606.855 & $4,68 \%$ & 364.050 & $6,09 \%$ \\
\hline Enfermedades de la piel y del tejido subcutáneo & 5.754 & $0,07 \%$ & 8.544 & $0,07 \%$ & 4.407 & $0,07 \%$ \\
\hline Enf. del sistema osteomuscular y del tejido conjuntivo & 21.969 & $0,27 \%$ & 31.618 & $0,24 \%$ & 17.015 & $0,28 \%$ \\
\hline Enfermedades del sistema genitourinario & 46.513 & $0,58 \%$ & 66.208 & $0,51 \%$ & 36.546 & $0,61 \%$ \\
\hline Embarazo, parto y puerperio & 4.285 & $0,05 \%$ & 7.199 & $0,06 \%$ & 2.850 & $0,05 \%$ \\
\hline Afecciones originadas en el periodo perinatal & 319.257 & $3,95 \%$ & 857.118 & $6,61 \%$ & 143.659 & $2,40 \%$ \\
\hline Malformaciones congénitas & 227.467 & $2,82 \%$ & 553.097 & $4,27 \%$ & 117.722 & $1,97 \%$ \\
\hline Causas externas & 1.782 .274 & $22,08 \%$ & 3.174 .503 & $24,48 \%$ & 1.241 .481 & $20,78 \%$ \\
\hline Todas las causas & 8.073 .466 & $100,00 \%$ & 12.965 .852 & $100,00 \%$ & 5.974 .265 & $100,00 \%$ \\
\hline
\end{tabular}

Escenario base: tasa anual de descuento (t.d) $=3 \%$; tasa anual de crecimiento de la productividad (t.p) $=1 \%$; Escenario 1 : t.d $=0 \%$, t.p $=2 \%$; Escenario 2 : t.d $=6 \%, \mathrm{t} . \mathrm{p}=0 \%$. Fuente: elaboración propia a partir del Registro de Defunciones por Causa de Muerte, Encuesta de Población Activa y la Encuesta de Estructura Salarial (año 2009) 
En el caso de las pérdidas de productividad como consecuencia de las baja laborales temporales ocasionadas por enfermedades y problemas de salud, la duración media ponderada de los procesos finalizados en el periodo ascendieron a 42,16 días y en total, se estimó en 268,515 millones el número de días correspondientes a situaciones de IT. La valoración monetaria de los días de trabajo perdidos como consecuencia de IT ascendió a 16.305 millones de euros. Los elementos más sobresalientes de la distribución en el número de días de baja, así como en la distribución de las pérdidas estimadas, fue el fuerte peso de las enfermedades del sistema osteomuscular y del tejido conjuntivo (un 18\% de los procesos de IT, un 27,75\% de los días de IT y un $27,97 \%$ de la pérdida estimada), seguidos por las causas externas (un 11,13\% de los procesos de IT, un $14,80 \%$ de los días de IT y un 14,73\% de la pérdida estimada) y por los trastornos mentales y del comportamiento (un 5,62\% de los procesos de IT, un 12,83\% de los días de IT y un 12,58\% de la pérdida estimada). Síntomas, signos y hallazgos anormales clínicos y de laboratorio acumuló un total de un 9,47\% de los procesos de IT, un 7,22\% de los días de IT y un 7,21\% de la pérdida estimada y las causas desconocidas o de difícil interpretación representaron el 10,94\% de los procesos de IT, un 8,64\% de los días de IT y un 8,67\% de la pérdida estimada. Asimismo, deben ser destacadas las enfermedades del sistema respiratorio, las cuales son responsables del 18,04\% de los procesos de IT, pero debido a su menor duración, representan un 5,31\% de los días de IT y un 5,18\% de la pérdida estimada.

Si acudimos a la lista reducida de enfermedades, 8 tipos de enfermedad reúnen el $46,2 \%$ de los casos y el 43,66\% de los días de baja laboral: colitis, enteritis, gastroenteritis infecciosas y diarreas; trastornos de la ansiedad, fobias sociales y trastornos depresivos; psicosis y episodios esquizofrénicos; resfriados comunes, bronquitis y faringitis; problemas de lumbago, estenosis espinal, trastorno de disco cervical y lesiones del hombro; situaciones de coma y estupor y trastornos del sueño; y la suma de los accidentes de tráfico y otros accidentes de transporte. 
Cuadro 3: Número de casos de IT e IP y pérdidas laborales por incapacidad laboral según la causa médica de la incapacidad.

\begin{tabular}{|c|c|c|c|c|c|c|c|}
\hline & $\begin{array}{c}\text { Casos de } \\
\text { IT } \\
\text { (Miles) }\end{array}$ & $\begin{array}{l}\text { Días en IT } \\
\text { (Millones) }\end{array}$ & $\begin{array}{c}\text { Millones } \\
\text { de } €\end{array}$ & $\begin{array}{c}\text { Casos } \\
\text { IPa } \\
\text { (Miles) }\end{array}$ & $\begin{array}{l}\text { Millones } \\
\text { de } €\end{array}$ & $\begin{array}{c}\text { Total } \\
\text { pérdidas } \\
\text { incapacidad }\end{array}$ & $\begin{array}{c}\% \\
\text { sobre } \\
\text { el total }\end{array}$ \\
\hline Enfermedades infecciosas y parasitarias & 541,3 & 5,62 & 329,9 & 22 & 541 & 870,9 & $2,50 \%$ \\
\hline Tumores & 73,9 & 8,39 & 530,3 & 58,7 & $1.442,60$ & $1.972,90$ & $5,70 \%$ \\
\hline Enf. de sangre y de órg. Hematopoyéticos & 10,2 & 0,76 & 46,8 & 2,9 & 72,1 & 119 & $0,30 \%$ \\
\hline Enfermedades endocrinas, nutricionales y metabólicas & 31,2 & 2,25 & 139,7 & 11,7 & 288,5 & 428,2 & $1,20 \%$ \\
\hline Trastornos mentales y del comportamiento & 357,9 & 34,49 & $2.050,90$ & 96,1 & $2.362,30$ & $4.413,20$ & $12,80 \%$ \\
\hline $\begin{array}{l}\text { Enf. del sistema nervioso y de los órganos de los } \\
\text { sentidos }\end{array}$ & 234,4 & 10,63 & 623 & 35,2 & 865,6 & $1.488,60$ & $4,30 \%$ \\
\hline Enfermedades del sistema circulatorio & 119,1 & 9,64 & 646,6 & 66 & $1.623,00$ & $2.269,60$ & $6,60 \%$ \\
\hline Enfermedades del sistema respiratorio & $1.148,90$ & 14,28 & 845,4 & 21,3 & 523 & $1.368,30$ & $4,00 \%$ \\
\hline Enfermedades del sistema digestivo & 354,7 & 10,51 & 668,8 & 19,8 & 486,9 & $1.155,60$ & $3,40 \%$ \\
\hline Enfermedades de la piel y del tejido subcutáneo & 73,9 & 2,49 & 151,7 & 5,9 & 144,3 & 295,9 & $0,90 \%$ \\
\hline Enf. del sistema osteomuscular y del tejido conjuntivo & $1.159,10$ & 74,56 & $4.560,50$ & 208,3 & $5.121,30$ & $9.681,80$ & $28,20 \%$ \\
\hline Enfermedades del sistema genitourinario & 122,9 & 5,08 & 299,3 & 11 & 270,5 & 569,8 & $1,70 \%$ \\
\hline Embarazo, parto y puerperio & 121 & 7,44 & 371,7 & 0,7 & 18 & $3^{89,7}$ & $1,10 \%$ \\
\hline Afecciones originadas en el periodo perinatal & 3,2 & 0,2 & 10,8 & 4,4 & 108,2 & 119 & $0,30 \%$ \\
\hline Malformaciones congénitas & 7,6 & 0,64 & 39,6 & 2,9 & 72,1 & 111,7 & $0,30 \%$ \\
\hline Síntomas, signos, NCOP. & 603,1 & 19,4 & $1.175,90$ & 54,3 & $1.334,40$ & $2.510,30$ & $7,30 \%$ \\
\hline Causas externas & 708,8 & 39,78 & $2.401,80$ & 62,3 & $1.532,80$ & $3.934,60$ & $11,50 \%$ \\
\hline Causa de la IT desconocido o de difícil interpretación & 696,7 & 23,21 & $1.413,00$ & 50,6 & $1.244,30$ & $2.657,20$ & $7,70 \%$ \\
\hline Todas las causas & $6.368,50$ & 268,71 & $16.305,50$ & 734,2 & $18.050,80$ & $34 \cdot 356,30$ & $100,00 \%$ \\
\hline
\end{tabular}

IT: incapacidad temporal; IP: incapacidad permanente; a En total se contabilizaron 919.946 personas en situación de IP durante 2009, los casos mostrados corresponden al resultado de ajustar según la tasa de empleo en los casos de incapacidad total y absoluta obtenida de la MCVL. 
Por otro lado, el número de pensiones contributivas de IP correspondiente al territorio nacional en el año 2009 fueron obtenidas del Anuario de Estadísticas Laborales y de Asuntos Sociales 2009, del Ministerio de Empleo y Seguridad Social. Se contabilizaron como media anual 32.178 prestaciones por Gran Invalidez, 384.492 por IP Absoluta, 503.276 por IP Total y 917 por IP Parcial. En total 919.946 personas en situación de IP. Como ya indicamos, en España las personas con la declaración de incapacidad parcial, absoluta y total pueden continuar recibiendo la prestación contributiva y desempañar un trabajo remunerado. Accedimos a la Muestra Continua de Vida Laboral (MCVL) para localizar las personas que aun teniendo una IP desempeñan un trabajo remunerado. Con esta información se calcularon las tasas de empleo de las personas que aun estando en situación de IP trabajaron al menos una vez durante el año 2009. Tras ajustar los casos de IP Absoluta e IP Total y excluir los casos de IP parcial, estimamos las pérdidas laborales asociadas a 734.225 casos de IP.

La valoración monetaria del tiempo de trabajo perdido como consecuencia de IP ascendió a 18.050 millones de euros. El grupo de enfermedades que mayores pérdidas por IP ocasionó fue el de enfermedades del sistema osteomuscular y del tejido conjuntivo (28\% del total de pérdidas y 208.311 casos), seguido de los trastornos mentales y del comportamiento ( $13 \%$ del total de pérdidas y 96.087 casos) y las enfermedades del sistema circulatorio (9\% del total de pérdidas y 66.014 casos). En suma, la pérdida de producción como consecuencia de la aparición de incapacidad laboral ascendió a 34.356 millones de euros, un $47 \%$ como consecuencia de la IT y un 52\% de la IP. En el caso de las bajas por IP no fue posible disponer de información sobre las causas reducidas de enfermedad que ocasionaron las bajas. 
Cuadro 4: Perdidas de productividad asociadas a enfermedades y problemas de salud según la causa médica responsable de la pérdida.

\begin{tabular}{|c|c|c|c|c|c|}
\hline & $\begin{array}{c}\text { Fallecimientos } \\
\text { prematuros }\end{array}$ & $\begin{array}{l}\text { Incapacidad } \\
\text { Temporal }\end{array}$ & $\begin{array}{l}\text { Incapacidad } \\
\text { Permanente }\end{array}$ & $\begin{array}{l}\text { Total } \\
\text { pérdidas }\end{array}$ & $\begin{array}{c}\text { \% sobre el } \\
\text { total de } \\
\text { pérdidas }\end{array}$ \\
\hline Enfermedades infecciosas y parasitarias & 365,7 & 329,9 & 541 & $1.236,60$ & $2,91 \%$ \\
\hline Tumores & $2.422,50$ & 530,3 & $1.442,60$ & $4 \cdot 395,40$ & $10,36 \%$ \\
\hline Enf. de sangre y de órg. Hematopoyéticos & 33,4 & 46,8 & 72,1 & 152,3 & $0,36 \%$ \\
\hline Enfermedades endocrinas, nutricionales y metabólicas & 134,1 & 139,7 & 288,5 & 562,3 & $1,33 \%$ \\
\hline Trastornos mentales y del comportamiento & 52,4 & $2.050,90$ & $2.362,30$ & $4.465,50$ & $10,52 \%$ \\
\hline Enf. del sistema nervioso y de los órganos de los sentidos & 261,6 & 623 & 865,6 & $1.750,20$ & $4,12 \%$ \\
\hline Enfermedades del sistema respiratorio & 383,9 & 845,4 & 523 & $1.752,20$ & $4,13 \%$ \\
\hline Enfermedades del sistema digestivo & 451,8 & 668,8 & 486,9 & $1.607,40$ & $3,79 \%$ \\
\hline Enfermedades de la piel y del tejido subcutáneo & 5,8 & 151,7 & 144,3 & 301,7 & $0,71 \%$ \\
\hline Enf. del sistema osteomuscular y del tejido conjuntivo & 22 & $4 \cdot 560,50$ & $5.121,30$ & $9.703,80$ & $22,87 \%$ \\
\hline Enfermedades del sistema genitourinario & 46,5 & 299,3 & 270,5 & 616,3 & $1,45 \%$ \\
\hline Embarazo, parto y puerperio & 4,3 & 371,7 & 18 & 394 & $0,93 \%$ \\
\hline Afecciones originadas en el periodo perinatal & 319,3 & 10,8 & 108,2 & 438,2 & $1,03 \%$ \\
\hline Malformaciones congénitas & 227,5 & 39,6 & 72,1 & 339,2 & $0,80 \%$ \\
\hline Causa desconocida o de difícil interpretación & - & $1.413,00$ & $1.244,30$ & $2.657,20$ & $6,26 \%$ \\
\hline Todas las causas & $8.073,50$ & $16.305,50$ & $18.050,80$ & $42.429,80$ & $100,00 \%$ \\
\hline
\end{tabular}

Unidades: millones de euros. Fuente: elaboración propia a través de diversas fuentes. 
La cifra total de pérdidas de producción estimada ascendió a 42.429,8 millones de euros, 8.073 millones de euros (19\%) como consecuencia de las muertes prematuras, 16.305 millones de euros (38\%) por la IT y 18.050 millones de euros (42\%) por la IP. El grupo de enfermedades que mayor impacto tuvo en términos de productividad perdida fue el de enfermedades del sistema osteomuscular y del tejido conjuntivo, con 9.703 millones de euros en total (23\% sobre el total), 22 millones de euros consecuencia de mortalidad prematura, 4.560 millones de euros por la IT causada y 5.121 millones de euros por la IP. Siendo la IP la causante de mayor pérdida de producción. El siguiente grupo de enfermedades con mayor impacto en términos de pérdidas de productividad fue las causas externas de mortalidad. En este caso, la IT causada por este grupo fue la responsable de que las causas externas de mortalidad sumaran el $13 \%$ de las pérdidas totales de producción (5.716 millones de euros). La pérdidas asociadas al grupo de tumores, son el tercer grupo de enfermedad con más impacto en términos de pérdidas de productividad. Es preciso señalar, cómo el elevado peso de las IT e IP ocasionadas por las enfermedades mentales y del comportamiento (4.413 millones de euros) superan las pérdidas de producción que ocasionaron los tumores por muertes prematuras (2.422 millones de euros).

Poniendo en contexto la cifra total de pérdida de producción estimada, las pérdidas laborales estimadas e imputables a enfermedades y problemas de salud sería el equivalente al 3,93\% del PIB español del año 2009. Asimismo, si acudimos a cifras oficiales del Ministerio de Sanidad, Servicios Sociales e Igualdad, nuestra cifra estimada de pérdidas laborales sería equivalente al 66,0\% del gasto sanitario público total de las CCAA y al $60,3 \%$ del total del gasto sanitario público del mismo año de referencia.

\section{Discusión y conclusiones}

El principal resultado del presente trabajo es la estimación de una pérdida laboral asociada a enfermedades y lesiones que ascendería a 42.430 millones de euros, de la cual 8.073 millones de euros corresponden a pérdidas derivadas de la mortalidad prematura, 18.050 millones de euros corresponden a pérdidas asociadas a situaciones de Incapacidad Permanente y 16.306 millones de euros a pérdidas asociadas a situaciones de Incapacidad Temporal. Ello supone un 3,93\% del Producto Interior Bruto de España o, equivalentemente, un 60,3\% del gasto sanitario público total del año 2009. Lógicamente, cuando comparamos las pérdidas totales estimadas con ciertos indicadores económicos como el PIB o el GSP, se debe tener en cuenta que no sería correcto interpretar una pérdida anualmente del $4 \%$ del PIB en concepto de pérdidas laborales asociadas a enfermedades o lesiones. Sin embargo, dada la magnitud de las cifras estimadas, los autores consideran que el PIB o GSP son los indicadores más adecuados para ser comparados con los resultados obtenidos para tener una aproximación comparable sobre el montante real que suponen las pérdidas laborales asociadas a enfermedades y lesiones en España.

En este trabajo, por primera vez se ha construido un mapa de las pérdidas laborales potenciales ocasionadas por enfermedades y lesiones. En trabajos previos (Oliva et al., 2012; Oliva-Moreno, 2012) se señalaba la importancia de las causas externas de muerte, tumores y enfermedades del sistema circulatorio en el caso de los fallecimientos prematuros, así como de las enfermedades osteomusculares y del sistema conjuntivo, los trastornos 
mentales y de la personalidad más las causas externas en el caso de las bajas laborales temporales. Con el presente trabajo, sumando la distribución de las pérdidas ocasionadas por las bajas permanentes (IP), las enfermedades osteomusculares, las causas externas, los trastornos mentales y los tumores quedan identificadas como las principales causas de pérdidas laborales. En general, los resultados obtenidos sobre la importante pérdida laboral estimada que suponen las enfermedades y lesiones van en concordancia con varios estudios donde estiman las pérdidas laborales ocasionadas por mortalidad prematura en enfermedades concretas tales como cáncer o enfermedades cardiovasculares, las cuales representan en torno al 20\% del coste total de la enfermedad (Gómez-de la Cámara et al., 2014; Hanly et at., 2014; Hanly \& Sharp, 2014; Leal et al., 2006).

Desafortunadamente, no podemos analizar los casos de IP por causas reducidas de enfermedad, pero con los datos de las muertes prematuras y los casos de IT ya se puede apuntar que la concentración de una parte tan elevada de carga de enfermedad y de pérdidas sociales en tan escaso número de enfermedades y lesiones debería estimular el diseño e implementación de políticas transversales de salud pública por parte de las autoridades públicas y también de responsables privados y civiles, dado que las potenciales ganancias (en términos de salud y en términos de pérdidas económicas evitables) son de una gran magnitud. Obviamente, el conocimiento científico sobre medidas de carácter preventivo es la condición previa para el diseño de este tipo de políticas. Por una parte, es evidente que los recursos no deben asignarse en función del impacto de una determinada enfermedad, sino allí donde una intervención produzca mayores beneficios en términos de salud y de ganancia de bienestar. Por otra, también resulta claro que el posible menú de actuaciones es muy amplio y que, por ello, el coste de oportunidad de destinar los recursos a aquellas actuaciones que no sean eficientes es muy elevado. Por tanto, el conocimiento de programas o políticas se convierten en una actividad clave para reducir los efectos negativos de las enfermedades y problemas de salud estudiados.

Otros aspectos sobre los que conviene reflexionar son las potenciales ventajas que puede aportar el trabajo multidisciplinar en los análisis de impacto de la enfermedad y en el diseño de programas y políticas. Este trabajo se ha beneficiado del análisis realizado por López et al. (2014), un equipo de investigación formado por profesionales del campo de la epidemiología y la salud pública. Dicho análisis nos ha permitido estudiar las pérdidas laborales asociadas a la IP por causa de enfermedad. La colaboración entre equipos de investigación multidisciplinares y la Administración Pública puede generar sinergias positivas para todas las partes implicadas y mejorar el contenido y la utilidad de los trabajos realizados al incorporar prismas complementarios que enriquezcan los análisis.

Por lo que respecta a la evolución de las cifras estimadas en este trabajo, si bien las pérdidas se han reducido en términos absolutos respecto a años anteriores, la caída del PIB del año 2009 ha supuesto que el peso relativo de las pérdidas sobre el PIB sea similar. En todo caso, se ha de señalar que (i) la evolución de las pérdidas a consecuencia de IP solo pueden cambiar en el largo plazo, dado que estamos estimando las pérdidas de casos acumulados; (ii) que en el caso de las pérdidas asociadas a IT la evolución del número de casos ha sido similar a la evolución de la población ocupada, si bien habrá que analizar la tendencia en los siguientes años de crisis en posteriores trabajos; (y iii) que en el caso de las pérdidas relacionadas con muertes prematuras sí se constata una evolución claramente favorable asociada a la mejora de los indicadores de salud. El análisis en detalle de la evolución de los fallecimientos prematuros puede encontrarse en Peña-Longobardo et al. (2015). 
La comparación de las cifras de pérdidas con el Gasto Sanitario permite poner en relación la cuantía de la pérdida laboral estimada en relación con los recursos que moviliza el sistema sanitario público en prevención y tratamiento de enfermedades y lesiones. Las pérdidas del año 2005 suponían el equivalente al 69,75\% del gasto sanitario público total consolidado (principio de devengo), las pérdidas del año 2007 suponían el equivalente al 69,49\% del gasto sanitario público total consolidado y, finalmente, las pérdidas estimadas para el año 2009 suponían el equivalente al 60,32\% del gasto sanitario público total consolidado del mismo año. Esta notable reducción observada en 2009, se debe al hecho de que el Gasto Sanitario Público representó una partida fuertemente inelástica en relación con la caída en el PIB. Es decir, el gasto sanitario siguió creciendo en el año 2009 frente a periodos anteriores, mientras las pérdidas de 2009, como ya señalamos se redujeron frente a las de 2007.

Frente a trabajos previos, hay líneas de avance ya señaladas: este es el primer trabajo donde se estima una distribución de las pérdidas de productividad por causa de enfermedad o lesión. No obstante, también existen otras limitaciones ya indicadas en trabajos previos pero que conviene recordar. En primer lugar, que las estimaciones de IT están basadas en los registros administrativos publicados o proporcionados por el INSS y, por tanto, puede existir una cierta infraestimación derivada de días de trabajo perdidos de corta duración que no llegan a ser contabilizados como baja laboral. Asimismo, en las cifras presentadas no se recogen todos los días de baja laboral de aquellos funcionarios públicos cubiertos por las aseguradoras de MUFACE, MUGEJU e ISFAS. Ello hace que la cifra real de pérdidas laborales por IT debería ser mayor a la estimada en el presente trabajo, pero no podemos aventurarnos a afirmar en qué grado. Tampoco contamos con herramientas para cuantificar posibles fraudes o dudas sobre la existencia de una causa médica real en las concesiones de IP e IT. Por tanto, se considera que en todas las concesiones existe un problema real (accidente, deterioro de la salud). Asimismo, al ajustar por tasa de empleo en el caso de personas en situación de IP se ha clasificado como empleada a toda persona que desempeñara una actividad laboral remunerada en el año de referencia, con independencia de si ésta era continuada o de corta duración. Ello supone aplicar un criterio de prudencia en la estimación, si bien también sugiere que la cifra estimada podría ser ligeramente superior si se introdujera un ajuste por duración de la actividad. Finalmente, dada la información proporcionada en las diferentes fuentes utilizadas, no ha sido posible estimar los costes derivados del llamado presentismo, esto es, la productividad laboral perdida derivada de la pérdida en la calidad del trabajo desempeñado como consecuencia de sufrir una determinada enfermedad o lesión.

Asimismo, se ha de subrayar que nuestro análisis se centra en las pérdidas de productividad laboral ocasionadas por enfermedad o lesiones en las personas directamente afectadas. No entramos a valorar otras pérdidas de bienestar, como serían las que se derivan del cuidado de las personas en situación de IT o de IP a consecuencia de una lesión grave por accidente o de un severo problema de salud discapacitante. Ello podría derivar en el internamiento en un centro de cuidado de larga duración de la persona afectada y derivar en un coste monetario familiar o público o bien ocasionar que la persona sea cuidada en su domicilio, bien contratando a un cuidador profesional o bien haciéndose cargo la propia familia de la persona (cuidados informales) o bien a cargo de un servicio público de atención a domicilio. Así, a las anteriores cifras, las enfermedades y lesiones ocasionan otros costes sociales que podríamos contabilizar a través de los costes 
del sistema de servicios sociales (o formales) a personas cuya autonomía se ha visto limitada (dependencia) y los costes soportados con cargo a recursos privados, así como otros recursos aportados por la red familiar (apoyo informal) (Oliva-Moreno et al., 2015).

Varias autoridades sanitarias españolas han mostrado recientemente interés en incorporar estudios de coste de la enfermedad como soporte a su toma de decisiones (Plan de Salud de Canarias 2004-2008; Estudios de Economía de la Salut, vol. I, II y III, Servèi Catalá de la Salut; varios años; Ministerio de Sanidad, varios años). Dicho interés sugiere que, una vez tomadas en cuenta las limitaciones de este tipo de estudios para la priorización de programas, y subrayando sus fortalezas a la hora de acercarnos al impacto social de las enfermedades, pueden ser una herramienta útil en el apoyo de la planificación de políticas públicas y, junto a otros indicadores de distintas dimensiones, pueden constituir una herramienta de valor para dar soporte a la toma de decisiones sobre asignación de recursos en políticas de salud, es decir, para construir Políticas Basadas en Pruebas («Evidence Based Policies»).

\section{Financiación y agradecimientos}

Este trabajo ha recibido una ayuda del Instituto de Estudios Fiscales para su elaboración. Asimismo, también se beneficia del apoyo del proyecto ECO2013-48217-C2-2-R «Impacto económico, sanitario y social de las enfermedades y los problemas de salud: información y herramientas para la evaluación de políticas públicas».

Los autores agradecen el apoyo institucional recibido del Instituto de Estudios Fiscales y el Instituto Nacional de la Seguridad Social (INSS) y dar las gracias a Angela Blanco y José María Labeaga (IEF) y María Dolores de Santa María y su equipo (INSS) por su ayuda y apoyo constante. También expresar nuestro agradecimiento por los comentarios recibidos de Javier Hernández y los participantes del taller realizado en el Instituto de Estudios Fiscales. Cualquier error u omisión en este estudio son de exclusiva responsabilidad de sus autores.

Los autores agradecen los comentarios y sugerencias constructivas recibidas por los revisores de este artículo.

\section{Referencias Bibliográficas}

Akobundu E, Ju J, Blatt L, \& CD, Mullins. (2006). Cost-of-illness studies : a review of current methods. Pharmacoeconomics, 24(9), 869-890.

Beck, E. J., Harling, G., Gerbase, S., \& DeLay, P. (2010). The cost of treatment and care for people living with HIV infection: implications of published studies, 1999-2008. Curr Opin HIV AIDS, 5(3), 215-224. doi: 10.1097/COH.obo13e32833860e9

Becker, G. (1964). The production of human capital. Chicago: Columbia University Press.

Blanchflower, D., \& Oswald, A. (2011). International happiness. http://www.nber.org/ papers/w16668 
Clabaugh, G, \& Ward, M. (2008). Cost-of-illness studies in the United States: a systematic review of methodologies used for direct cost. Value in Health, 11(1), 13-21.

Departament de Salut. Servèi Catalá de la Salut. Estudios de Economía de la Salut, vol. I, II y III, Generalitat de Cataluña, varios años.

Ferrer-i-Carbonell, A. (2013). Happiness economics. SERIES, 4, 35-60.

Gómez-de la Cámara A, Pinilla-Domínguez P, Vázquez-Fernández Del Pozo S, García Pérez L, Rubio-Herrera MA, Gómez-Gerique JA, Gutiérrez-Fuentes JA, Rivero-Cuadrado A, Serrano-Aguilar P. (2014). Costs resulting from premature mortality due to cardiovascular causes: A 20-year follow-up of the DRECE study. Rev Clin Esp. pii: Soo14-2565(14)00247-1

Graham, C. (2008). Happiness and Health: Lessons-And Questions-For Public Policy. $27,72-87$.

Grossman, M. (1972a). The Demand for Health: A Theoretical and Empirical Investigation. New York: Columbia University Press.

Grossman, M. (1972b). On the concept of health capital and the demanda for health. Journal of Political Economy, 8o(2), 223-255.

Grossman, M. (2000). The human capital model of the demand for health. In J. P. Newhouse \& A. J. Culyer (Eds.), Handbook of Health Economics. Amsterdam: North-Holland-Springer-Verlag.

Hanly P, Soerjomataram I, Sharp L. (2014). Measuring the societal burden of cancer: The cost of lost productivity due to premature cancer-related mortality in Europe. Int $J$ Cancer. doi: 10.1002/ijc.29105. [Epub ahead of print] PMID: 25066804

Hanly PA, Sharp L. (2014). The cost of lost productivity due to premature cancer-related mortality: an economic measure of the cancer burden. BMC Cancer. 14:224. doi: 10.1186/1471-2407-14-224. PMID: 24670067

Hodgson, T., \& Meiners, M. (1982). Cost-of-Illness Methodology: A Guide to Current Practices and Procedures. The Milbank Memorial Fund Quarterly. Health and Society, 6o(3), 429-462.

Hoefman, R. J., van Exel, J., \& Brouwer, W. (2013). How to include informal care in economic evaluations. Pharmacoeconomics, 31(12), 1105-1119. doi: 10.1007/ S40273-013-0104-Z

Hu, TW. (2006). Perspectives: an international review of the national cost estimates of mental illness, 1990-2003. J Ment Health Policy Econ, 9(1), 3-13.

INE. (2009a). Encuesta de Estructura Salarial. http://www.ine.es/jaxi/menu.do?type=pcaxis\&path=/t22/p133\&file=inebase $\& \mathrm{~L}=0$

INE. (2009b). Encuesta de Población Activa. http://www.ine.es/inebaseDYN/epa30308/epa_inicio.htm 
INE. (2009c). Regitro de defunciones según la causa de la muerte. http://www.ine.es/jaxi/menu.do?type $=$ pcaxis\&path $=/ \mathrm{t} 15 / \mathrm{p} 417 \&$ file $=$ inebase\&L $=0$

Krol, M., Brouwer, W., \& Rutten, F. F. (2013). Productivity costs in economic evaluations: past, present, future., 31, 537-540.

Leal J, Luengo-Fernandez R, Gray A, Petersen S, Rayner M. (2006). Economic burden of cardiovascular diseases in the enlarged European Union. European heart journal. 27(13): 1610-9.

Lopez, M. A., Duran, X., Alonso, J., Martinez, J. M., Espallargues, M., \& Benavides, F. G. (2014). Estimación de la carga de enfermedad por incapacidad laboral permanente en España durante el período 2009-2012. Revista espanola de salud publica, 88(3), 349-358. doi: 10.4321/s1135-57272014000300005

Max, W., Rice, D., \& MacKenzie, E. (1990). The Lifetime Cost of Injury. Inquiry, 27(4), 332-343.

Minister of Public Works and Government Services Canada. (2002). Economic Burden of Illness in Canada. http://publications.gc.ca/collections/Collection/H21-1361998E.pdf

Ministerio de Empleo y Seguridad Social. (2009). Anuario de estadísticas del ministerio de empleo y seguridad social. http://www.empleo.gob.es/es/estadisticas/ contenidos/anuario.htm

National Heart Lung and Blood Institute. (2012). Fact Book. Fiscal Year 2012. http:// www.nhlbi.nih.gov/about/documents/factbook/2012

Ng, C. S., Lee, J. Y., Toh, M. P., \& Ko, Y. (2014). Cost-of-illness studies of diabetes mellitus: a systematic review. Diabetes Res Clin Pract, 105(2), 151-163. doi: 10.1016/j. diabres.2014.03.020

Oliva, J., \& Aranda-Reneo, I. (2012). Pérdidas laborales ocasionadas por las enfermedades, lesiones y problemas de salud durante el año 2007. Presupuesto y Gasto Público, $68,157-176$.

Oliva-Moreno, J. (2012). Loss of labour productivity caused by disease and health problems: what is the magnitude of its effect on Spain's Economy? The European Journal Of Health Economics, 13(5), 605-614. doi: 10.1007/s10198-011-0344-9

Oliva-Moreno, J., Peña-Longobardo, L.M, \& Vilaplana, C. (2015). An estimation of the value of informal care provided to dependent people in Spain.

Peña-Longobardo, L.M, Aranda-Reneo, I, Oliva-Moreno, J., \& Vall-Castello, J. (2015). Pérdidas laborales ocasionadas por los fallecimientos prematuros. un análisis del periodo 2005-2009. Revista Española de Salud Pública, (en prensa).

Sachs, J.D. (2001). Macroeconomics and health: Investing in health for economic development. Report of the Commission on macroeconomics and health. World Health Organization. http://whqlibdoc.who.int/publications/2001/924154550x.pdf 
Servicio Canario de la Salud. Plan de Salud de Canarias 2004-2008. Consejería de Sanidad del Gobierno de Canarias. Gobierno de Canarias.

Suhrcke, M., Mckee, M., Sauto-Arce, R., Tsolova, S., \& Mortensen, J. (2006). The contribution of health to the economy in the European Union. Public Health, 120(11), 994-1001.

Withrow, D., \& Alter, D. A. (2011). The economic burden of obesity worldwide: a systematic review of the direct costs of obesity. Obes Rev, 12(2), 131-141. doi: 10.1111/j.1467789X.2009.00712.x 